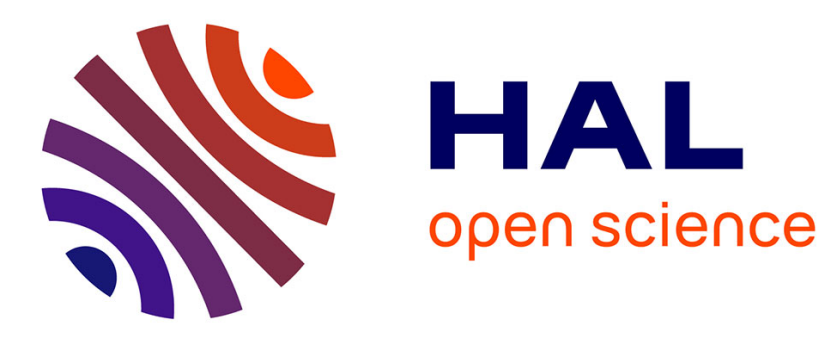

\title{
FFLO-wave-vector Lock-in Effect in Quasi-1D Superconductors
}

Mihail D. Croitoru, Alexandre I. Buzdin

\section{To cite this version:}

Mihail D. Croitoru, Alexandre I. Buzdin. FFLO-wave-vector Lock-in Effect in Quasi-1D Superconductors. Journal of Superconductivity and Novel Magnetism, 2015, 28 (4), pp.1305-1308. 10.1007/s10948014-2910-8 . hal-01155491

\section{HAL Id: hal-01155491 \\ https://hal.science/hal-01155491}

Submitted on 26 May 2015

HAL is a multi-disciplinary open access archive for the deposit and dissemination of scientific research documents, whether they are published or not. The documents may come from teaching and research institutions in France or abroad, or from public or private research centers.
L'archive ouverte pluridisciplinaire HAL, est destinée au dépôt et à la diffusion de documents scientifiques de niveau recherche, publiés ou non, émanant des établissements d'enseignement et de recherche français ou étrangers, des laboratoires publics ou privés. 


\title{
FFLO-wave-vector Lock-in Effect in Quasi-1D Superconductors
}

\author{
M. D. Croitoru • A. I. Buzdin
}

\begin{abstract}
We study the phase transition into the FuldeFerrell-Larkin-Ovchinnikov state in high magnetic field in quasi-one dimensional superconductors within the quasiclassical formalism, taking into account the interchain Josephson coupling and the paramagnetic spin splitting. We show that anomalies in the field-direction dependence of the upper critical field when the magnetic field length equals to the FFLO period, previously described in [29], are characterized by the lock-in effect of the FFLO modulation wave vector, which is governed by the magnetic length.
\end{abstract}

\section{Introduction}

The effect of high magnetic fields on the superconducting state in compounds with reduced dimensionality has stirred up one of the main research interests in the field of superconductivity in the last few decades [1,2]. This has been motivated, in particular by discoveries of superconducting high crystallographic quasi-one dimensional structures with unique properties in magnetic field, e.g., the quasi1D organic Bechgaard salts (TMTSF) ${ }_{2} \mathrm{X}$, where anion $\mathrm{X}$ is $\mathrm{PF}_{6}, \mathrm{ClO}_{4}$, etc. [1-8] polysulfur nitride $\left(\mathrm{SN}_{x}\right)$ [9], the metal-chalcogenide-based compounds [10-15], transition

M. D. Croitoru $(\varangle) \cdot$ A. I. Buzdin

Université de Bordeaux, LOMA, UMR

5798, F-33400 Talence, France

e-mail: mikhail.croitoru@uni-bayreuth.de

\section{D. Croitoru}

Departement Fysica, Universiteit Antwerpen,

Groenenborgerlaan 171, B-2020

Antwerpen, Belgium

\author{
A. I. Buzdin \\ Institut Universitaire de France, Paris France
}

metal carbides [16], the quasi-1D $M_{2} \mathrm{Mo}_{6} \mathrm{Se}_{6}$ compounds [17-19], lithium purple bronze [20], and arrays of 4 Ångstrom superconducting carbon nanotubes embedded in the linear pores channels of AFI zeolite single crystals $[21,22]$. A very large upper critical fields, exceeding the Pauli paramagnetic limit, for a magnetic field aligned parallel to their most conducting layers in the these materials as well as the NMR measurements and the anomalous in-plane anisotropy of the upper critical field [3, 23] for field parallel to the conducting chains, e.g., in the organic compound (TMTSF) ${ }_{2} \mathrm{ClO}_{4}$ favor the existence of FuldeFerrell-Larkin-Ovchinnikov (FFLO) superconducting phase with a spin singlet pairing $[24,25]$.

In $[23,26]$, it has been demonstrated that the FFLO modulation strongly interferes with the orbital effect and provides the main source of the critical field anisotropy. Our subsequent analysis of the properties of the quasilow dimensional superconductors revealed that in some of them the resonance between the period of the FFLO modulation and the period of the interlayer coupling modulation induced by the external field is possible [27-29]. The obtained results predict the anomalous cusps in the temperature and angular dependencies of the in-plane critical field. Their experimental observation may serve as a direct evidence for the appearance of the FFLO phase in quasi-1D and quasi-2D superconductors [26-29]. This peculiar behavior of the in-plane anisotropy of the FFLO phase completes the anomalous oscillatory out-of-plane angular dependence of the critical field in FFLO state [30-34].

In the studies of the orbital corrections to the upper critical field in the FFLO phase, we assumed that the absolute value of the FFLO wave vector is determined in the pure Pauli limit. However, the orbital contribution also influences the absolute value of the FFLO modulation vector. One can expect that the orbital correction can modify the absolute 
value of the FFLO wave vector especially at resonances. To clarify this issue in this work, we investigate influence of the orbital motion on the FFLO characteristics in quasi-1D superconductors.

\section{The Model}

We consider a quasi-one-dimensional (quasi-1D) conductor with the following electron spectrum

$E_{\mathbf{p}}=\frac{p_{x}^{2}}{2 m_{x}}+2 t_{y} \cos \left(p_{y} d_{y}\right)+2 t_{z} \cos \left(p_{z} d_{z}\right)$

where $d_{y}$ and $d_{z}$ are the interchain distances along the $y$ - and $z$-axis, respectively. We assume that the couplings between chains in both directions are small [see Fig. 1], i.e., $t_{z} \ll T_{c 0}$ and $t_{y} \ll T_{c 0}$, but sufficiently large to suppress the CDW and SDW transitions, to stabilize the superconducting long-range order and to make the mean field treatment justified, $T_{c 0}^{2} / E_{F} \ll t_{z}, T_{c 0}^{2} / E_{F} \ll t_{y}$. Here, $T_{c 0}$ is the critical temperature of the system at $H=0$.

We choose the gauge, for which the vector potential of the external magnetic field is $\mathbf{A}=[\mathbf{H} \times \mathbf{r}][[\mathbf{r}=(x, 0,0)]$ is a coordinate along the $x$-chain], i.e., $A_{z}=-x H_{y}=$ $-x H \sin \alpha \sin \theta, A_{y}=x H_{z}=x H \cos \alpha \sin \theta, A_{x}=0$, where $H$ is the amplitude of the magnetic field and $\alpha$ accounts for its direction from the positive $z$-axis in the $y-z$ plane, while $\theta$ is the angle accounting the field direction from the positive $x$-axis. Taking into account that the system is near the second-order phase transition, we can employ the linearized Eilenberger equation for a quasi-1D superconductor in the presence of the magnetic field (in the momentum representation with respect to the coordinate $z$ ) [35]:

$\left(\Omega_{n}+\widehat{\Pi}\right) f_{\omega}\left(x, y, p_{z}\right)=\Delta(x, y) \operatorname{sign}\left(\omega_{n}\right)$

with $\widehat{\Pi} \equiv \frac{\hbar}{2} v_{F} \frac{\partial}{\partial x}+2 i t_{z} \sin \left(p_{z} d_{z}\right) \sin \left(Q_{x} x\right)$, where we have assumed that the vector potential varies slowly at the interchain distances (this assumption means that we neglect the diamagnetic screening currents and take the magnetic field as uniform and given by the external field, $H$ ), and for the purpose of simplicity we have chosen the

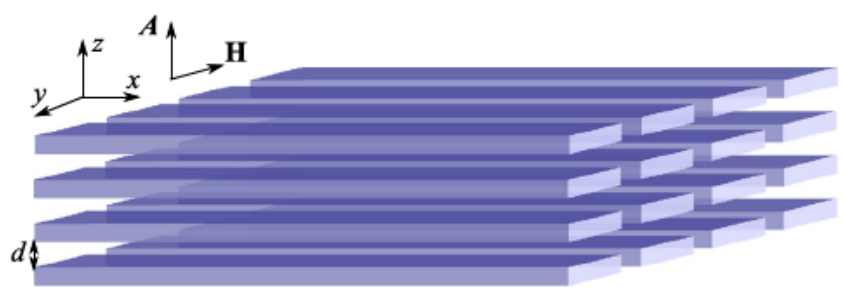

Fig. 1 Scheme of the quasi-1D conductor in a parallel magnetic field external magnetic field in the $x-y$ plane. Here, $Q_{x}=$ $-\pi d_{z} H_{y} / \phi_{0}=-\pi d_{z} H \sin \alpha \sin \theta / \phi_{0}$ with $\phi_{0}=\pi \hbar c / e$, $h=\mu_{B} H$ is the Zeeman energy, $\mathbf{v}_{F}=v_{F_{x}} \mathbf{i}$ is the Fermi velocity along the $x$-axis, and $\Omega_{n} \equiv \omega_{n}-i h \operatorname{sign}\left(\omega_{n}\right)$. Usually, a quasi-1D superconductor can be considered as a system in the clean limit, meaning that the mean free path is much larger than the corresponding intra-chain coherence length, $\xi_{0}^{x}=v_{F x} /\left(2 \pi T_{c 0}\right)$. The order parameter is defined self-consistently as

$\frac{1}{\lambda} \Delta(x, y)=2 \pi T \operatorname{Re} \sum_{\omega>0}\left\langle f_{\omega}\left(x, y, p_{z}\right)\right\rangle$,

where $\lambda$ is the pairing constant and the brackets denote averaging over $p_{y}$ and $p_{z}$ and $v_{F_{x}}$,

$\langle\ldots\rangle \equiv N^{(1)} \int_{-\frac{\pi}{d y}}^{\frac{\pi}{d y}} \frac{d_{y} d p_{y}}{2 \pi} \int_{-\frac{\pi}{d z}}^{\frac{\pi}{d z}} \frac{d_{z} d p_{z}}{2 \pi} \frac{1}{2} \sum_{ \pm v_{F}}(\ldots)$

with $N^{(1)} \equiv m / 2 \pi p_{x} d_{y} d_{z}$. The temperature unit is so that the Boltzmann constant $k_{B}=1$. We seek the solution of the linearized Eilenberger equation (2) in the form

$f_{\omega}\left(x, y, p_{z}\right)=e^{i \mathbf{q r}} \sum_{m} e^{i m \mathbf{Q} \cdot \mathbf{r}} f_{m}\left(\omega_{n}, p_{z}\right)$.

Because of the form for $f_{\omega}\left(x, y, p_{z}\right)$ of (5) one can write $\Delta(x, y)$ as

$\Delta(x, y)=e^{i \mathbf{q r}} \sum_{m} e^{i 2 m \mathbf{Q} \cdot \mathbf{r}} \Delta_{2 m}\left(k_{z}\right)$.

From symmetry considerations, it follows that $\Delta_{-2 m}=$ $\Delta_{2 m}$. Adopting a second-order approximation in the small parameter $t_{z} / T_{c 0}$ to the solution of (2) and substituting (5) and (6) back into (2), and making use of the definition for the order parameter function, (3-4), we obtain two systems of coupled equations $[36,37]$

$$
\begin{aligned}
\Delta_{0}\left[P+t_{z}^{2} b_{0}\right] & =t_{z}^{2}\left[g_{\mp 0} \Delta_{\mp 2}+g_{ \pm 0} \Delta_{ \pm 2}\right], \\
\Delta_{+2}\left[P+\delta_{ \pm 2}+t_{z}^{2} b_{ \pm 2}\right] & =t_{z}^{2}\left[g_{ \pm 0} \Delta_{0}+g_{ \pm 2} \Delta_{ \pm 4}\right], \\
\Delta_{-2}\left[P+\delta_{\mp 2}+t_{z}^{2} b_{\mp 2}\right] & =t_{z}^{2} g_{\mp 0} \Delta_{0} \\
\Delta_{+4}\left[P+\delta_{ \pm 4}+t_{z}^{2} b_{ \pm 4}\right] & =t_{z}^{2} g_{ \pm 2} \Delta_{ \pm 2},
\end{aligned}
$$

where

$$
\begin{aligned}
& \delta_{\xi n}=\frac{\pi T}{2} \sum_{\omega, \pm} \frac{1}{B_{0}^{ \pm}}-\frac{1}{B_{4 \xi}^{ \pm}}, \\
& b_{\xi n}=\frac{\pi T}{2} \sum_{\omega, \zeta, \pm} \frac{1}{B_{n \xi}^{ \pm 2}\left[B_{n \xi}^{ \pm} \pm i \hbar v_{F} \zeta Q_{x} / 2\right]}, \\
& g_{\xi n}=\frac{\pi T}{2} \sum_{\omega, \pm} \frac{1}{B_{0}^{ \pm} B_{1 \xi}^{ \pm} B_{2 \xi}^{ \pm}},
\end{aligned}
$$


where $B_{n \xi}^{ \pm} \equiv \Omega_{n} \pm i \hbar v_{F}\left(q_{x}+\xi n Q_{x}\right) / 2$ with $\xi=$ $0, \pm 1$. In (7), the following notations are introduced: $P=$ $\Delta T_{c P} / A_{c} T_{c}$ with $A_{c}=1-\frac{h}{T_{c P}} \frac{\partial T_{c P}}{\partial h}$ and $\Delta T_{c P}=T_{c}-T_{c P}$. Here, $T_{c P}$ is the superconducting onset temperature in the pure Pauli limit determined by

$\ln \left(\frac{T_{c 0}}{T_{c P}}\right)=\pi T_{c P} \sum_{n} \frac{1}{\omega_{n}}-\frac{1}{2} \sum_{ \pm}\left(\Omega_{n} \pm i \hbar v_{F} q_{x} / 2\right)^{-1}$.

The solution of the system of (7) is given by

$T_{c}=T_{c P}\left[1-A_{c}\left(S_{\mathrm{O}}+S_{\mathrm{R}}\right)\right]$

with the "orbital" term $S_{\mathrm{O}} \equiv t_{z}^{2} b_{0}(\mathbf{q}, \mathbf{Q})$ and the "resonance" term $S_{\mathrm{R}}(\mathbf{q}, \mathbf{Q})=\min _{\xi} S_{\mathrm{R}}^{\xi}(\mathbf{q}, \mathbf{Q})$ with

$$
\begin{aligned}
S_{\mathrm{R}}^{\xi}(\mathbf{q}, \mathbf{Q}) \equiv & -\frac{\left(\bar{a}_{\xi}-\bar{b}_{\xi}\right) t_{z}^{2}-\delta_{\xi}}{2} \\
& -\frac{t_{z}^{2}}{2} \sqrt{\left[\bar{a}_{\xi}-\bar{b}_{\xi}-\delta_{\xi} / t_{z}^{2}\right]^{2}+4 g_{1 \xi}^{2}},
\end{aligned}
$$

where

$\bar{a}_{\xi}=b_{0}-\frac{g_{-\xi 0}^{2} t_{z}^{2}}{\delta_{-\xi 2}}, \quad \bar{b}_{\xi}=b_{\xi}-\frac{g_{ \pm 2}^{2} t_{z}^{2}}{\delta_{\xi 4}}$

and those values of $\xi= \pm$ are chosen that maximize the critical temperature. The FFLO modulation vector $\mathbf{q}$ corresponds to the maximum of $T_{c}(\mathbf{q})$, and its direction is along $x$-axis. For the out-of-resonance conditions [27], $\Delta_{ \pm 2}=0$ and the solution is just $T_{c}=T_{c P} /\left[1+A_{c} S_{\mathrm{O}}\right]$ [29]. For the in-resonance conditions $\delta_{\xi}=0$ and $S_{\mathrm{R}}^{\xi}(\mathbf{q}, \mathbf{Q})=-c_{\xi} t_{z}^{2}$. The resonance occurs when $|\mathbf{q} \pm 2 \mathbf{Q}|=|\mathbf{q}|$, i.e., $q=$ $\pm Q$. As was indicated in [27], the developed formalism valid only for the field orientation satisfying the following relation $\sin \theta \gg t_{z} \phi_{0} / \pi \hbar d H v_{F}$.

\section{Results and Discussions}

For the numerical calculations, we restrict ourselves to the following parameters: $d_{x}=0.339 \mathrm{~nm}, d_{y}=1.283 \mathrm{~nm}$, $d_{z}=1.539 \mathrm{~nm}$ [12]. The superconducting temperature was chosen to be $T_{c 0}=6.5 \mathrm{~K}$ [13], and the band couplings are $t_{z}=0.65 \mathrm{~K} \sim t_{y}$ and $t_{z}=2.25 \mathrm{~K}$. Introducing the dimensionless Fermi velocity parameter, $\eta=$ $\pi \hbar d_{z} v_{F} / \phi_{0} \mu_{B}=\hbar v_{F} Q_{x} / \mu_{B}$. In this work, we consider $v_{F}=1.2 \times 10^{5} \mathrm{~m} / \mathrm{sec}$, which corresponds to $\eta=3.16$. The summation over the Matsubara frequencies was performed numerically.

Figure 2 illustrates the temperature dependence of the absolute value of the FFLO modulation wave vector $q$ calculated without accounting for the orbital motion (red solid line), when accounting the orbital effect with $\Delta_{ \pm 2}=0$ (green dashed line), and with $\Delta_{ \pm 2} \neq 0$ (green solid line) when calculated in the clean limit. The blue dashed line
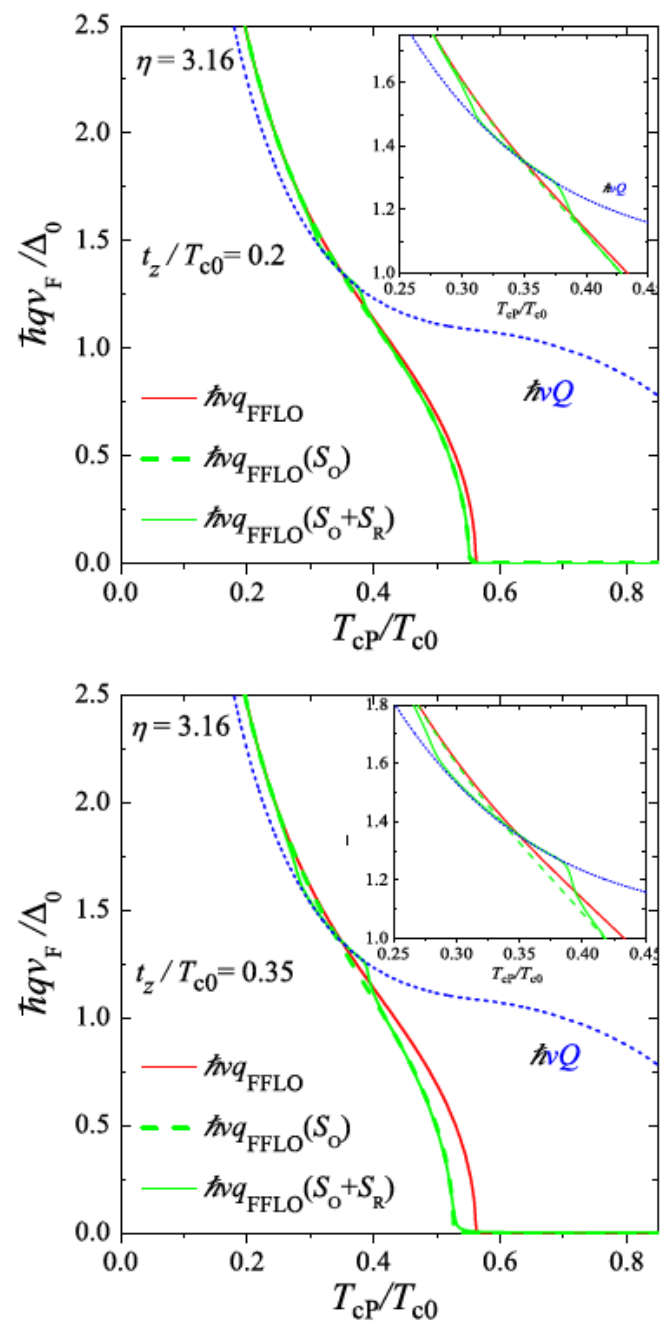

Fig. 2 The absolute value of the FFLO modulation vector $q$ versus of $T_{c P} / T_{c 0}$ for an s-wave quasi-1D superconductor when accounting for the orbital effects within the first iteration. The upper panel: $t_{c} / T_{c}=$ 0.2 . The lower panel: $t_{c} / T_{c}=0.35$. Solid line takes into account the influence of resonance. Short dashed line shows the field vector $Q$

gives the upper critical field in the Pauli limit in terms of the magnetic wave vector $\mathbf{Q}$. The direction of the external magnetic field is characterized by $\theta$, the angle the applied field $\mathbf{H}$ makes from the principal $x$-axis of the quasi-1D compound and, in this particular case, is fixed to $\theta=35$ degrees. The optimal direction of the modulation vector is along the conductive chains for $0<T<T^{*}$ with $T^{*} \simeq 0.56 T_{c 0}$ for the clean sample in the Pauli limit. The upper critical field in the inhomogeneous superconducting state diverges at $T \rightarrow 0$ and appreciably higher than the paramagnetic limit already for $T_{c P} / T_{c 0}<0.3$ [38]. As seen from the figure, the magnitude of the $\mathbf{q}$ vector monotonically increases from zero at the tricritical point until infinity. At $T_{c P} / T_{c 0} \approx 0.35$, the FFLO wave vector $q$ intersects the magnetic wave vectors $\mathbf{Q}$ and the resonance anomaly in the superconducting characteristics occurs [27]. 
We clearly see that the orbital contribution influences the absolute value of the FFLO modulation vector. The influence of the orbital effect on the modulation vector is rather for $t_{z}=1.30 \mathrm{~K}$ except for the region in the close proximity to the resonance. In the vicinity of the resonance, where the unperturb $q$ and $Q$ curves intersect, appears an interesting lock-in effect: while sweeping the $T_{c P} / T_{c 0}$ across the resonance the FFLO vector jumps from its almost unperturb value $q_{0}$, determined without the orbital contribution, to the magnetic field vector $Q$, crosses the resonance and then jumps back on the original $q_{0}$ curve. For the case $t_{z}=$ $2.25 \mathrm{~K}$, the contribution of the orbital effect is seen already near the tricritical point, which is shifted to the lower value due to the orbital contributions even for $\Delta_{ \pm 2}=0$.

In conclusion, we have described the behavior of the FFLO modulation wave vector in quasi-1D superconductors in high magnetic field with different hopping parameters. We demonstrated that at the resonance the interplay between the orbital and paramagnetic effects may result in a lockin effect between modulation wave vector and the magnetic wave vector. This effect may become an additional tool to evidence the FFLO state. In our study, we have assumed $s$ wave pairing; however, the model can be easily extended to the $d$-wave pairing [28]. We expect that all the obtained results will remain qualitatively similar.

Acknowledgments We thank D. Jerome for useful discussions. We acknowledge the support by the French ANR program "ElectroVortex" and European NanoSC COST Action MP1201. M.D.C. acknowledges the support by the BELSPO Return to Belgium Grant.

\section{References}

1. Jerome, D., Schulz, H.J.: Adv. Phys. 31, 299 (1982)

2. Buzdin, A.I., Bulaevskii, L.N.: Sov. Phys. Usp. 27, 830 (1984). Usp. Fiz. Nauk 144, 415 (1984)

3. Yonezawa, S., Kusaba, S., Maeno, Y., Auban-Senzier, P., Pasquier, C., Bechgaard, K., Jérome, D.: Phys. Rev. Lett. 100, 117002 (2008)

4. Shinagawa, J., Kurosaki, Y., Zhang, F., Parker, C., Brown, S.E., Jérome, D., Bechgaard, K., Christensen, J.B.: Phys. Rev. Lett. 98, 147002 (2007)

5. Lee, I.J., Naughton, M.J., Danner, G.M., Chaikin, P.M.: Phys. Rev. Lett. 78, 3555 (1997)

6. Lee, I.J., Chaikin, P.M., Naughton, M.J.: Phys. Rev. B 62, R14669 (2000)

7. Lee, I.J., Chaikin, P.M., Naughton, M.J.: Phys. Rev. B 65, 180502(R) (2002)

8. Oh, J.I., Naughton, M.J.: Phys. Rev. Lett. 92, 067001 (2004)

9. Lou, L.F.: J. Appl. Phys. 66, 979 (1989)
10. Srivastava, S., Avasthi, B.N.: J. Mater. Sci. 27, 3693 (1992)

11. Lu, Y.F., Takayama, T., Bangura, A.F., Katsura, Y., Hashizume, D., Takagi, H.: arXiv:1308.3766

12. Zhang, Q.R., Rhodes, D., Zeng, B., Besara, T., Siegrist, T., Johannes, M.D., Balicas, L.: Phys. Rev. B 88, 024508 (2013)

13. Zhang, Q., Li, G., Rhodes, D., Kiswandhi, A., Besara, T., Zeng, B., Sun, J., Siegrist, T., Johannes, M.D., Balicas, L.: Sci. Rep. 3, 1446 (2013)

14. Niu, C.Q., Yang, J.H., Li, Y.K., Chen, B., Zhou, N., Chen, J., Jiang, L.L., Chen, B., Yang, X.X., Cao, C., Dai, J., Xu, X.: Phys. Rev. B 88 (2013)

15. Singh, D.J.: Rev, Phys. B 88, 174508 (2013)

16. Scherer, W., Hauf, Ch., Presnitz, M., Scheidt, E.-W., Eickerling, G., Eyert, V., Hoffmann, R.-D., Rodewald, U.C., Hammerschmidt, A., Vogt, Ch., Pöttgen, R. Angew. Chem. Int. Ed. 49, 1578. (2010); G. Eickerling, Ch. Hauf, E.-W. Scheidt, L. Reichardt, Ch. Schneider, A. Muñ oz, S. Lopez-Moreno, A. H. Romero, F. Porcher, G. André, R. Pöttgen, and W. Scherer, Z. Anorg. Allg. Chem. 639, 1985, 2013

17. Brusetti, R., Briggs, A., Laborde, O., Potel, M., Gougeon, P.: Phys. Rev. B 49, 8931 (1994)

18. Petrović, A.P., Lortz, R., Santi, G., Decroux, M., Monnard, H., Fischer, Ø., Boeri, L., Andersen, O.K., Kortus, J., Salloum, D., Gougeon, P., Potel, M.: Phys. Rev. B 82, 235128 (2010)

19. Bergk, B., Petrović, A.P., Wang, Z., Wang, Y., Salloum, D., Gougeon, P., Potel, M., Lortz, R.: New J. Phys. 13, 103018 (2011)

20. Mercure, J.-F., Bangura, A.F., Xiaofeng, X.u., Wakeham, N., Carrington, A., Walmsley, P., Greenblatt, M., Hussey, N.E.: Phys. Rev. Lett. 108, 187003 (2012)

21. Tang, Z.K., Zhang, L.Y., Wang, N., Zhang, X.X., Wen, G.H., Li, G.D., Wang, J.N., Chan, C.T., Sheng, P.: Science 292, 2462 (2001)

22. Wang, Z., Shi, W., Xie, H., Zhang, T., Wang, N., Tang, Z.K., Zhang, X.X., Lortz, R., Sheng, P., Sheikin, I., Demuer, A.: Phys. Rev. B 81, 174530 (2010)

23. Croitoru, M.D., Houzet, M., Buzdin, A.I.: Phys. Rev. Lett. 108, 207005 (2012)

24. Larkin, A.I., Ovchinnikov, Yu.N.: Zh. Eksp. Teor. Phys. 47, 1136 (1964). Sov. Phys. JETP 20, 762 (1965)

25. Fulde, P., Ferrell, R.A.: Phys. Rev. 135, A550 (1964)

26. Croitoru, M.D., Houzet, M., Buzdin, A.I., Supercond, J.: Nov. Magn. 25, 1283 (2012)

27. Croitoru, M.D., Buzdin, A.I.: Phys. Rev. B 86, 064507 (2012)

28. Croitoru, M.D., Buzdin, A.I.: J. Phys.: Condens. Matter. 25, 125702 (2013)

29. Croitoru, M.D., Buzdin, A.I.: Phys. Rev. B 86, 224508 (2012)

30. Houzet, M., Buzdin, A.: EuroPhys. Lett. 50, 375 (2000)

31. Buzdin, A.I., Kulic, M.: J. Low Temp. Phys. 54, 203 (1984)

32. Buzdin, A.I., Brison, J.P.: EuroPhys. Lett. 35, 707 (1996)

33. Buzdin, A.I., Brison, J.P.: Phys. Lett. A 218, 359 (1996)

34. Brison, J.P., Keller, N., Lejay, P., Huxley, A.D., Schmidt, L., Buzdin, S., Bernhoeft, N., Mineev, V.P., Stepanov, A.N., Flouquet, J., Jaccard, D., Julian, S., Lonzarich, G.: Phys. B 199, 70 (1994)

35. Kopnin, N.B.: Theory of Nonequilibrium Superconductivity. Clarendon Press, Oxford (2001)

36. Croitoru, M.D., Buzdin, A.I., Supercond, J.: Nov. Magn. 26, 1657 (2013)

37. Croitoru, M.D., Buzdin, A.I.: Phys. Rev. B 89, 224506 (2014)

38. Buzdin, A.I., Polonskii, S.V.: JETP 66, 422 (1987). Zh. Eksp. Teor. Fiz. 93, 747 (1987) 